\title{
5. Improving Indigenous health: Are mainstream determinants sufficient?
}

\author{
Nicholas Biddle
}

The headline target of the Council of Australian Governments 'closing the gap' agenda is the elimination of the difference between Indigenous and non-Indigenous life expectancy in Australia. While this in and of itself is a worthwhile (if difficult to achieve) aim, life expectancy represents just one aspect of physical and mental health. Instead, the World Health Organization (WHO 2006) defines health as 'not only the absence of infirmity and disease but also a state of physical, mental and social wellbeing'.

This definition of health makes it clear that individuals can be completely free of disease and appear in a physical sense to be healthy but, because of low mental or social wellbeing, be quite unhealthy. Similarly, a person may have one or a number of chronic conditions but, because of a supportive family or community, consider themselves to be quite healthy.

Indigenous Australians extend the definition of health even further and also use a much broader definition of health than the absence of disease. In 1990, the National Aboriginal Health Strategy defined health as follows: 'Health does not just mean the physical wellbeing of the individual but refers to the social, emotional, spiritual and cultural wellbeing of the whole community' (National Health and Medical Research Council (NHMRC) 1996, cited in Jackson and Ward 1999). That is, not only is one's own wellbeing important, but so too is the wellbeing of the community in which one lives and has ongoing attachment. This definition is not without problems and may, according to Boddington and Raisanen (2009) be 'so broad in compass it may with some justification be said not to be a definition of health at all, but of something far more encompassing'.

Notwithstanding this complicated relationship between physical health and wellbeing, previous research has demonstrated a clear empirical link between physical health and subjective wellbeing. For example, in Kahneman and Deaton (2010), having a health condition was found to be negatively associated with emotional wellbeing and life satisfaction. What's more, the size of the association was quite large - similar in magnitude to marital status and income. Unlike changes in income (Shields, Wheatley Price and Wooden 2009), however, 
individuals only tend to experience partial adaptation to disability (Oswald and Powdthavee 2008). That is, even after a number of years, individuals who become disabled reported lower levels of life satisfaction than previously.

Analysis has also shown that reporting relatively low levels of subjective wellbeing was associated with worse self-assessed health into the future (Siahpush, Spittal and Singh 2008). That is, not only does physical health determine emotional wellbeing and life satisfaction, but it is also determined by it. The link between mental health and subjective wellbeing (and emotional wellbeing in particular) is even clearer. Operationally, the question often used to identify negative affect (or periods of intense feelings of sadness) often comes from the module on psychological distress and either the Kessler-5 (K5) or Kessler-10 (K10) scale (Australian Bureau of Statistics (ABS) 2010). Scores on these scales are used as predictors of mental health-related conditions and are, empirically, negatively correlated with positive measures of emotional wellbeing (like happiness) and life satisfaction. Nonetheless, mental health and wellbeing are distinct concepts, as noted by the Social Health Reference Group (2004), with those with mental health conditions often able to obtain high levels of wellbeing (provided they receive sufficient support in doing so).

Indigenous Australians report lower levels of physical health than the nonIndigenous population. At around 22 per cent, Indigenous Australians were around twice as likely to report that their health was either fair or poor compared to non-Indigenous Australians (ABS/Australian Institute of Health and Welfare (AIHW) 2010). Although Indigenous adults were found to be only slightly less likely to have a long-term condition than non-Indigenous adults (based on analysis of the 2004-05 National Aboriginal and Torres Strait Islander Health Survey (NATSIHS)), the difference was much greater when the relatively young Indigenous age profile is taken into account. When age-standardised, Indigenous Australians in 2004-05 were 1.6 times as likely to report that they had asthma, 3.4 times as likely to report diabetes/high sugar levels and 10.0 times as likely to report a kidney disease.

There is a large literature on the determinants of Indigenous health. However, most of the empirical analysis has tended to focus on reasonably narrow definitions of health and narrowly defined determinants. This is not surprising, because the focus of much of the analysis is on explaining the difference between Indigenous and non-Indigenous Australians. Explaining differences between the two populations necessitates using data that contains both populations and measures that are applicable to both. The aim of this paper is to summarise new research on the determinants of Indigenous health using data from the 2008 National Aboriginal and Torres Strait Islander Social Survey (NATSISS). Before then, I summarise some of the available literature on the determinants of Indigenous health. 


\section{The determinants of Indigenous health}

There is a considerable amount of research on the physical health of Indigenous Australians in general, and the determinants of the gap between Indigenous and non-Indigenous Australians in particular. Firstly, Indigenous Australians are more likely to live in remote and very remote Australia than the non-Indigenous population - areas where access to health services can be difficult. Indigenous Australians need to travel greater distances to access primary health services and, even more so, hospitals and other expensive health procedures (ABS/ AIHW 2010).

Secondly, Indigenous Australians start off with worse health outcomes than the non-Indigenous population. They are more likely to be born prematurely and to have low birth weight (ABS/AIHW 2010), due in part to the fact that Indigenous mothers are much more likely to be relatively young and to smoke and/or drink alcohol during pregnancy. Indigenous Australians also experience worse physical health outcomes through childhood and into adolescence. They are less likely to have been breastfed up until 12 months, have a worse diet, were less likely to be vaccinated at a young age, more likely to be exposed to passive smoking, more likely to have a long term health condition, and more likely to have been hospitalised due to illness and/or injury (ABS/AIHW 2010).

Research in other contexts clearly demonstrates a link between childhood physical and mental health and later health outcomes. For example, Blackwell, Hayward and Crimmins (2001: 1280) conclude that:

...respondents who experienced childhood health problems were more likely to experience a variety of chronic illnesses and conditions such as cancer, lung illnesses, cardiovascular conditions, and arthritis/ rheumatism.

Results from the Aboriginal Birth Cohort study are beginning to show this link for a cohort of Aboriginal children born between January 1987 and March 1990 in Darwin (Sayers et al. 2011). One of the explanations for poor adult physical health amongst Indigenous Australians is likely to be poor child and adolescent health.

The socioeconomic status of Indigenous children is one of the main determinants of their poor physical health status. However, socioeconomic status also has an effect on health throughout the lifecourse. The association between an individual's social and economic status and their health has long been established (Matthews, Jagger and Hancock 2006; Wilkinson and Marmot 2003). In the Indigenous context, Biddle (2006) showed that not only were there high returns to education for Indigenous health, but that the difference between Indigenous and non-Indigenous Australians in terms of education outcomes explained a 
large component of the gap in self-assessed and physical health. Using a greater range of socioeconomic variables, Booth and Carroll (2008) found that economic factors explained around half of the gap in self-assessed health between Indigenous and non-Indigenous Australians.

The link between socioeconomic status and health is not unique to the Indigenous population. However, there are other factors that in the Australian context at least are unique to the Indigenous population. First is a history of dispossession of land and exclusion from citizenship rights. Much of the social determinants of health literature focuses on the negative health effects of having a lack of control of one's life (Wilkinson and Marmot 2003). Given the importance of land to Indigenous culture and the historic removal of Indigenous Australians from many parts of the Australian continent (Broome 2010), it is quite likely that this has had long-lasting effects on physical and mental health.

Related to the dispossession of Indigenous land is the denigration of Indigenous culture within Australia through much of its history since European colonisation. Since at least the mid 1800s, Indigenous Australians were seen as part of a 'dying race' that would either be incorporated into the non-Indigenous population through intermarriage or die out through excess mortality or low fertility (Smith 1980). The clearest expression of this is the 'stolen generations' or Indigenous children who were forcibly removed from their natural families due to their indigeneity (Broome 2010). As will be seen later in this chapter, this is still having ongoing health effects on those who were removed and on their families.

A final potentially negative effect on health is ongoing discrimination in Australia towards Indigenous Australians and racism in everyday life. Discrimination refers to:

...a situation in which persons ... who are equally productive in a physical or material sense are treated unequally in a way that is related to an observable characteristic such as race, ethnicity or gender (Altonji and Blank 1999: 3168).

According to Dunn et al. (2004: 411), this includes 'a belief in a racial hierarchy, in racial separation and in "race" itself' as well as new forms of racism like intolerance towards specific cultural groups.

Discrimination is very difficult to detect using standard data sources, however there is evidence that an Indigenous Australian with the same level of education and experience as a non-Indigenous Australian has a lower probability of employment and a lower income (Hunter 2004). While it is not possible to control for unobservable characteristics in the data used in Hunter (2004), experimental data would suggest that a person with a recognisably Indigenous name is less likely to be treated favourably in the labour market than someone with a name not identifiable as being Indigenous (Booth, Leigh and Varganova 2010). 
Regardless of whether a person is actually discriminated against, in terms of health effects it is perhaps equally important whether they themselves perceive that they experienced discrimination. Biddle (2011a) showed that Indigenous Australians were significantly and substantially more likely to report that they were discriminated against compared to non-Indigenous Australians both when applying for a job and also in their current job (in terms of seeking promotion and other employment conditions).

Despite the potential negative effects on Indigenous health just discussed, there are also a number of positive aspects identified in the literature. While the pressures on Indigenous culture have been cited as a potential determinant of ill health, it should always be kept in mind that the converse of this is that those who are able to maintain key aspects of Indigenous culture and heritage are likely to benefit from the protective health benefits that cultural participation can bring. Related to this, Rowley et al. (2008) found relatively low morbidity and mortality in a remote Northern Territory Indigenous community (compared to the rest of the Territory). The authors speculated that the ability to maintain a healthy lifestyle and the ability to maintain control over their own culture was a key explanation for this positive health effect.

\section{Relationship between self-assessed health and other measures of wellbeing}

Previous analysis (Biddle 2006; Booth and Carroll 2008) has shown that even after controlling for a wide range of socioeconomic variables, Indigenous Australians are more likely to report their health as being fair or poor than the non-Indigenous population. Although this analysis gives some indication as to what the determinants of self-assessed health are in general, they did not give any indication as to whether these determinants held, and what the size of the association is for the Indigenous population in isolation. Furthermore, it is not possible with datasets like the NATSIHS to look at Indigenous-specific determinants of health like cultural/language maintenance or discrimination.

In order to test for Indigenous-specific determinants of health, Table 5.1 presents summary statistics and Table 5.2 results from econometric analysis of the 2008 NATSISS. The dependent variable is the probability of reporting one's health as being fair or poor. ${ }^{1}$

1 The main conclusions from the analysis hold when using all five self-assessed health categories and estimating using the ordered probit model. However, it is more difficult to interpret the size of the association using ordered probit so the simpler binary probit model is used. 
Survey Analysis for Indigenous Policy in Australia

Table 5.1 Explanatory variables assumed to be associated with selfassessed health, Indigenous Australians, 2008

\begin{tabular}{|c|c|}
\hline Explanatory variables & Proportion \\
\hline Female & 0.568 \\
\hline Aged $15-24$ & 0.250 \\
\hline Aged 25-34 & 0.231 \\
\hline Aged 55 plus & 0.162 \\
\hline Aged 15-24, female & 0.138 \\
\hline Aged 25-34, female & 0.139 \\
\hline Aged 55 plus, female & 0.093 \\
\hline Lives in remote Australia & 0.337 \\
\hline Not married & 0.540 \\
\hline Lives in a couple family with children & 0.332 \\
\hline Lives in a couple family with no children but dependents & 0.077 \\
\hline Lives in a single parent family with children & 0.194 \\
\hline Lives in a single parent family with no children but dependents & 0.079 \\
\hline Lives in an 'other' family type & 0.161 \\
\hline Has a non-Indigenous person living in the household & 0.363 \\
\hline Main language spoken at home is not English & 0.147 \\
\hline Changed usual residents in the previous five years & 0.607 \\
\hline Not in the labour force & 0.398 \\
\hline Unemployed & 0.093 \\
\hline Works part-time & 0.195 \\
\hline Occupation is neither a manager or a professional & 0.407 \\
\hline Main job is in the CDEP scheme & 0.062 \\
\hline Completed Year 10 or 11 only & 0.445 \\
\hline Completed Year 9 or less & 0.364 \\
\hline Has a degree or higher as highest qualification & 0.052 \\
\hline Has a diploma as highest qualifications & 0.047 \\
\hline Has a certificate as highest qualification & 0.222 \\
\hline Victim of physical or threatened violence in previous 12 months & 0.254 \\
\hline Arrested in previous five years & 0.152 \\
\hline Lives in a house that does not meet the occupancy standard & 0.204 \\
\hline Speaks, understands or is learning an Indigenous language & 0.295 \\
\hline Involved in cultural events, ceremonies or organisations in the previous 12 months & 0.647 \\
\hline Able to have a say on important issues within the community all or most of the time & 0.272 \\
\hline Strongly agrees or agrees that in general people can be trusted & 0.357 \\
\hline Did not run out of money for basic living expenses in the last 12 months & 0.717 \\
\hline Feels very safe or safe at home after dark & 0.788 \\
\hline Did not report any neighbourhood or community problems & 0.233 \\
\hline Felt discriminated against in the previous 12 months due to Indigenous status & 0.276 \\
\hline Was removed from natural family & 0.097 \\
\hline A relative was removed from their natural family & 0.416 \\
\hline
\end{tabular}


One of the limitations of the 2008 NATSISS for analysing the determinants of health is the focus on self-reported health measures. However, the subjective nature of self-assessed health is also one of its strengths. By focusing on how an individual actually feels (rather than how one might expect them to feel based on objective characteristics) it is possible to obtain more accurate predictions of future behaviour. According to Clark, Frijters and Shields (2008: 119; referring to Kahneman et al. 1993):

...[m]any panel studies have found that subjective wellbeing at time $\mathrm{t}$ predicts future behaviour, in that individuals clearly choose to discontinue activities associated with low levels of wellbeing.

Results in Table 5.2 are presented across two models. Model 1 is very similar to previous analysis with the explanatory variables being standard demographic and socioeconomic measures. In Model 2, on the other hand, a range of Indigenous-specific variables are used, representing different aspects of Indigenous language and cultural maintenance, financial stress, feelings of safety, neighbour or community problems, discrimination and forcible removal from one's natural family.

Table 5.2 Factors associated with self-assessed health, Indigenous Australians, 2008

\begin{tabular}{|c|c|c|c|c|}
\hline Explanatory variables & Mode & & Model & 2 \\
\hline Female & -0.022 & & -0.047 & $* *$ \\
\hline Aged $15-24$ & -0.168 & $* * *$ & -0.324 & $* * *$ \\
\hline Aged 25-34 & -0.101 & $* * *$ & -0.172 & $* * *$ \\
\hline Aged 55 plus & 0.037 & & 0.079 & $* * *$ \\
\hline Aged $15-24$, female & 0.069 & ** & 0.089 & $* *$ \\
\hline Aged 25-34, female & -0.012 & & -0.005 & \\
\hline Aged 55 plus, female & -0.022 & & -0.010 & \\
\hline Lives in remote Australia & -0.053 & $* * *$ & -0.057 & $* * *$ \\
\hline Not married & -0.042 & $* *$ & -0.058 & * \\
\hline Lives in a couple family with children & -0.045 & $* * *$ & -0.057 & $* *$ \\
\hline Lives in a couple family with no children but dependents & -0.020 & & -0.002 & \\
\hline Lives in a single parent family with children & 0.006 & & -0.011 & \\
\hline $\begin{array}{l}\text { Lives in a single parent family with no children but } \\
\text { dependents }\end{array}$ & 0.069 & ** & 0.070 & * \\
\hline Lives in an 'other' family type & 0.071 & $* *$ & 0.071 & * \\
\hline Has a non-Indigenous person living in the household & -0.003 & & 0.007 & \\
\hline Main language spoken at home is not English & -0.041 & ** & -0.039 & \\
\hline Changed usual residents in the previous five years & 0.010 & & -0.009 & \\
\hline Not in the labour force & 0.248 & $* * *$ & 0.223 & $* * *$ \\
\hline
\end{tabular}




\begin{tabular}{|c|c|c|c|c|}
\hline Explanatory variables & Model & & Model & 2 \\
\hline Unemployed & 0.146 & *** & 0.104 & *** \\
\hline Works part-time & 0.066 & *** & 0.067 & $* * *$ \\
\hline Occupation is neither a manager or a professional & -0.008 & & -0.017 & \\
\hline Main job is in the CDEP scheme & 0.027 & & 0.050 & \\
\hline Completed Year 10 or 11 only & 0.007 & & -0.005 & \\
\hline Completed Year 9 or less & 0.079 & $* * *$ & 0.078 & $* * *$ \\
\hline Has a degree or higher as highest qualification & 0.001 & & -0.017 & \\
\hline Has a diploma as highest qualifications & -0.007 & & -0.030 & \\
\hline Has a certificate as highest qualification & 0.016 & & 0.012 & \\
\hline $\begin{array}{l}\text { Victim of physical or threatened violence in previous } 12 \\
\text { months }\end{array}$ & & & 0.025 & \\
\hline Arrested in previous five years & & & 0.042 & ** \\
\hline $\begin{array}{l}\text { Lives in a house that does not meet the occupancy } \\
\text { standard }\end{array}$ & & & -0.012 & \\
\hline $\begin{array}{l}\text { Speaks, understands or is learning an Indigenous } \\
\text { language }\end{array}$ & & & 0.003 & \\
\hline $\begin{array}{l}\text { Involved in cultural events, ceremonies or organisations in } \\
\text { the previous } 12 \text { months }\end{array}$ & & & -0.028 & * \\
\hline $\begin{array}{l}\text { Able to have a say on important issues within the } \\
\text { community all or most of the time }\end{array}$ & & & -0.030 & * \\
\hline $\begin{array}{l}\text { Strongly agrees or agrees that in general people can be } \\
\text { trusted }\end{array}$ & & & -0.052 & *** \\
\hline $\begin{array}{l}\text { Did not run out of money for basic living expenses in the } \\
\text { last } 12 \text { months }\end{array}$ & & & -0.101 & $* * *$ \\
\hline Feels very safe or safe at home after dark & & & -0.081 & *** \\
\hline Did not report any neighbourhood or community problems & & & -0.068 & *** \\
\hline $\begin{array}{l}\text { Felt discriminated against in the previous } 12 \text { months due } \\
\text { to Indigenous status }\end{array}$ & & & 0.030 & * \\
\hline Was removed from natural family & & & 0.063 & *** \\
\hline A relative was removed from their natural family & & & 0.044 & $* * *$ \\
\hline Probability of the base case & 0.216 & & 0.537 & \\
\hline Pseudo R-Squared & 0.1287 & & 0.1468 & \\
\hline Number of observations & 7536 & & 7240 & \\
\hline
\end{tabular}

Notes: The base case individual for all estimations is: male; aged 35-54; lives in non-remote Australia; is married; lives in a couple family without children with Indigenous Australians only in the household; speaks English at home and did not change usual residence in the previous 5 years; employed full-time as a manager or professional outside of the CDEP scheme; has completed Year 12; and does not have a qualification.

*** Marginal effect for which the coefficient is statistically significant at the $1 \%$ level of significance.

** Marginal effect for which the coefficient is statistically significant at the $5 \%$ level of significance.

${ }^{*}$ Marginal effect for which the coefficient is statistically significant at the $10 \%$ level of significance.

Source: Customised calculations using the 2008 NATSISS 
There are a number of policy-relevant results contained in Model 1. Indigenous Australians in remote Australia are less likely to report their health as being fair or poor compared to those in non-remote Australia. This may reflect language differences in interpreting the question with Sibthorpe, Anderson and Cunningham (2001) noting the limitations of a global self-assessed health measure when applied to all Indigenous populations regardless of language background. However, it is important to note that the results hold after controlling for whether or not the person speaks a language other than English at home or when estimating on those who speak English only. Taking the results at face value, however, analysis of the 2008 NATSISS would tend to support the findings in Rowley et al. (2008) that living in a remote area has a protective effect on health.

Being out of the labour force was associated with poorer health on average. There is, however, a strong potential that having poor health leads to opting out of the labour force rather than the other way around. At the very least, the causal influence is likely to run in both directions. However, those who are unemployed are, by definition, actively seeking work and willing to commence work if the opportunity arises. The fact that they are also significantly and substantially more likely to report that they had fair or poor health suggests that for Indigenous Australians, employment status does impact on health.

It is important to note that those whose main job was in the Community Development Employment Program (CDEP) scheme did not have a significantly different probability of reporting fair or poor health than those in mainstream employment. Furthermore, using a separate (but unreported) test, those in the CDEP scheme were less likely to report fair or poor health than those who were unemployed, the other natural comparison group. To the extent that the CDEP scheme provided an alternative to unemployment benefits in certain communities in 2008, the analysis in Table 5.2 gives circumstantial evidence of a positive effect on health. This supports the finding in Biddle (2011b) that Indigenous Australians in the CDEP scheme reported higher subjective wellbeing (happiness and sadness) than those who were unemployed.

There was a non-linear association between education and health. Those who had completed Year 9 or less had a significantly and substantially higher probability of reporting fair or poor health than the base case (those who had completed Year 12) and, based on a separate test, those who had completed Year 10 or 11. However, there was no difference between those who had completed Year 10 or 11 and those who had completed Year 12, nor was there any association with post-school qualifications. This supports the finding in Biddle (2011b) that, in terms of wellbeing, lower levels of education are of greatest importance for Indigenous Australians. 
The results in Model 1 generally represent mainstream determinants of health. Estimating the associations for the Indigenous population separately shows how these determinants manifest for the Indigenous population, although there is nothing particularly Indigenous-specific about them. The additional variables included in Model 2, however, show that Indigenous-specific variables are important in explaining variation within the Indigenous population in terms of self-assessed health. While there was no association with Indigenous language maintenance, results presented in Table 5.2 showed a small association between involvement in Indigenous culture. Furthermore, those who felt they were able to have a say on important issues and those with high levels of generalised trust were less likely to have fair or poor health, as were those who did not experience financial stress (through running out of money) and those who felt safe at home.

One cannot be too confident about the direction of causality in the above Indigenous-specific variables. It is quite possible that one's health affects these variables as much as being affected by them. However, they do give an indication that cultural maintenance and broader notions of community wellbeing have a strong interaction with health. It would really only be with an Indigenousspecific longitudinal dataset that the causal direction could be established (a point returned to in the concluding section).

Given the way the variables are defined, it is much more likely that the last three variables are affecting health rather than being affected by it. Specifically, those who were discriminated against were more likely to report fair or poor health as were those who were removed from their natural family and those who had relatives removed. While this is likely to represent a causal influence, it is a little difficult to identify specific policies that stem from these results. On the one hand, most forms of discrimination based on Indigenous status are illegal in Australia. However, more than one-quarter of the population reported that they felt discriminated against, so clearly policies alone are not sufficient in this area. Furthermore, the policy of forcibly removing children from their family is a historical rather than a current one. This notwithstanding, the results do demonstrate the potentially long-term effects of such policies, and do support to a certain extent calls for compensation by those who were affected.

\section{Concluding comments}

The determinants of Indigenous health are likely to interact in complex ways, with no single factor completely dominating. Those Indigenous Australians who start off with poor health are likely to find it relatively difficult to complete education, achieve stable employment and receive a decent income. Furthermore, mainstream education completion has been shown to enhance rather than act as 
a barrier to Indigenous cultural participation - a further determinant of broad notions of health. Despite this complexity, there exists a rich range of data sets that can be used to show the size and strength of the association between potential health risk factors and physical or self-assessed health, including the 2008 NATSISS. While it is not possible to conclusively test for the effect of particular policies, nor is it possible to establish causal relationships, the analysis of associations at least hints at policy priorities.

Ultimately, what the results presented in this paper show is that focusing on mainstream determinants of health (like those in the Closing the Gap agenda) will quite possibly lead to improvements in health. Increasing Indigenous education is likely to extend Indigenous life expectancy, as will improving access to employment opportunities. However, it will not be enough to close the gap entirely. Until Indigenous Australians no longer feel they are discriminated against and until a complete rapprochement with the stolen generations takes place, gaps between Indigenous and non-Indigenous Australians in health outcomes are likely to remain.

Furthermore, results presented in this paper would suggest that it does matter how policies on Closing the Gap are pursued. Indigenous Australians living in remote Australia have better self-assessed health (on average) than those in non-remote parts of the country, after controlling for other characteristics. If Indigenous Australians in remote areas are forced or even encouraged to move to other parts of the country in an attempt to improve employment and education, then there may be countervailing effects on health. Furthermore, removal of the CDEP scheme is a current aim of government policy. I have not seen any evidence that convincingly links having a CDEP scheme available in an area to Indigenous Australians opting out of mainstream employment. It is doubtful, therefore, that removing the scheme will in and of itself improve access to mainstream employment. It would appear from results in this paper though that the scheme (as it was structured in 2008) may have a protective effect on health. Removing the CDEP scheme and moving Indigenous Australians onto unemployment benefits will quite likely worsen Indigenous health with debatable effects on non-CDEP employment.

The results presented in this paper were all based on cross-sectional data. While this analysis provides useful insights, the strength of the policy conclusions are seriously constrained. However, there are no specific datasets with a large enough Indigenous sample and enough Indigenous-specific questions to be able to accurately identify the factors that influence Indigenous health. For example, it is not possible to test whether moving into or out of employment in general - or the CDEP scheme in particular - changes one's health. It may well be that those who are currently employed have other unobserved characteristics that influence their health. Alternatively, it is not possible to show whether changes 
in cultural participation influence health. Perhaps more importantly, it is not possible to analyse or even control for the effect of child health on later physical or mental health.

The Closing the Gap agenda on life expectancy is generational. There is still time in 2011 to develop the evidence base that will enable governments to achieve this aim. A part of this evidence base is analysis of the NATSISS (or the NATSIHS). However, this alone will not be enough. Proper randomised controlled trials that test for the causal effect of specific policies is a part of this process. So too is a longitudinal survey specifically designed for the Indigenous population that contains data across the lifecourse. With this in mind, I renew the call for a National Closing the Gap Survey (first raised in Biddle and Yap 2010) that would provide an annual tracking of progress in achieving the Closing the Gap targets, and allow the development of a more robust evidence base to support Indigenous policy in Australia by allowing researchers to ask 'what influences Indigenous health', rather than 'what is associated with Indigenous health'.

\section{References}

Altonji, J. G. and Blank, R. M. 1999. 'Race and gender in the labor market', in O. Ashenfelter and D. Card (eds), Handbook of Labor Economics, Elsevier, North Holland.

Australian Bureau of Statistics (ABS) 2010. National Aboriginal and Torres Strait Islander Social Survey: Users' Guide, 2008, cat. no. 4720.0, ABS, Canberra.

_ /Australian Institute of Health and Welfare (ABS/AIHW) 2010. The Health and Welfare of Australia's Aboriginal and Torres StraitIslander Peoples 2008, cat. no. 4704.0, ABS, Canberra.

Biddle, N. 2006. 'The association between health and education in Australia: Indigenous/non-Indigenous comparisons', The Economic and Labour Relations Review, 17 (1): 107-42.

201 la. 'Income, work and Indigenous livelihoods', Lecture 5, Measures of Indigenous Wellbeing and Their Determinants Across the Lifecourse, 2011 CAEPR Lecture Series, CAEPR, ANU, Canberra.

2011 b. 'Measuring and analysing wellbeing', Lecture 2, Measures of Indigenous Wellbeing and Their Determinants Across the Lifecourse, 2011 CAEPR Lecture Series, CAEPR, ANU, Canberra. 
and Yap, M. 2010. Demographic and Socioeconomic Outcomes Across the Indigenous Australian Lifecourse: Evidence from the 2006 Census, CAEPR Research Monograph No. 31, ANU E Press, Canberra.

Blackwell, D. L., Hayward, M. D. and Crimmins, E. M. 2001. 'Does childhood health affect chronic morbidity in later life?', Social Science and Medicine, 52: 1269-84.

Boddington P, and Räisänen U. 2009. 'Theoretical and practical issues in the definition of health: Insights from Aboriginal Australia', Journal of Medicine and Philosophy, 34: 49-67

Booth, A. and Carroll, N. 2008. 'Economic status and the Indigenous/nonIndigenous health gap', Economics Letters, 99 (3): 604-6.

, Leigh, A. and Varganova, E. 2010. 'Does racial and ethnic discrimination vary across minority groups? Evidence from a field experiment', CEPR Discussion Paper No. DP7913, Centre for Economic Policy Research, London.

Broome, R. 2010. Aboriginal Australians: A History Since 1788, Allen and Unwin, Sydney.

Clark, A. E., Frijters, P. and Shields, M. 2008. 'Relative income, happiness and utility: An explanation for the Easterlin paradox and other puzzles', Journal of Economic Literature, 46: 95-144.

Dunn, K. M., Forrest, J., Burnley, I. and McDonald, A. 2004. 'Constructing racism in Australia', Australian Journal of Social Issues, 39 (4): 409-30.

Hunter, B. H. 2004. Indigenous Australians in the Contemporary Labour Market, cat. no. 2052.0, ABS, Canberra.

Jackson, L. R. and Ward, J. E. 1999. 'Aboriginal health: Why is reconciliation necessary?', Medical Journal of Australia, 170: 437-40.

Kahneman, D. and Deaton, A. 2010. 'High income improves evaluation of life but not emotional well-being', Proceedings of the National Academy of Science, 107 (38): 16489-93.

, Frerickson, B. L., Schreiber, C. A. and Redelmeier, D. A. 1993. 'When more pain is preferred to less: Adding a better end', Psychological Science, 4 (6): 401-5.

Matthews, R., Jagger, C. and Hancock, R. 2006. 'Does socio-economic advantage lead to a longer, healthier old age?', Social Science and Medicine, 62 (10): 2489-99. 
National Health and Medical Research Council (NHMRC) 1996. 'Promoting the health of Indigenous Australians. A review of infrastructure support for Aboriginal and Torres Strait Islander health advancement. Final report and recommendations', NHMRC, Canberra.

Oswald, A. J. and Poddthavee, N. 2008. 'Does happiness adapt? A longitudinal study of disability with implications for economists and judges', Journal of Public Economics, 92 (5-6): 1061-77.

Rowley, K. G., O’Dea, I., Anderson, R., McDermott, K., Saraswati, K., Tilmouth, R., Roberts, I. F. J., Zaiman, W., Jenkins, A., Best, J. D., Wang, Z. and Brown, A. 2008. 'Lower than expected morbidity and mortality for an Australian Aboriginal population: 10-year follow-up in a decentralised community', The Medical Journal of Australia, 188 (5): 283-87.

Sayers, S., Davison, B., Fitz, J. and Singh, G. 2011. 'Aboriginal Birth Cohort Study-Wave 3: Selected results: The good news and the bad news', Aboriginal and Islander Health Worker Journal, 35 (5): 23-4.

Shields, M. A., Wheatley Price, S. and Wooden, M. 2009. 'Life satisfaction and the economic and social characteristics of neighbourhoods', Journal of Population Economics, 22 (2): 421-43.

Siahpush, M., Spittal, M. and Singh, G. K. 2008. 'Happiness and life satisfaction prospectively predict self-rated health, physical health, and the presence of limiting, long-term health conditions', American Journal of Health Promotion, 23 (1): 18-26.

Sibthorpe, B., Anderson, I. and Cunningham, J. 2001. 'Self-assessed health among indigenous Australians: How valid is a global question?', American Journal of PublicHealth, 91 (10): 1660-3.

Smith, L. R. 1980. The Aboriginal Population of Australia, ANU Press, Canberra.

Social Health Reference Group 2004. 'Social and emotional well being framework: a national strategic framework for Aboriginal and Torres Strait Islander mental health and social and emotional well being 2004-2009', Australian Government, Canberra, viewed 4 July 2012, available at <http://www.atns. net.au/agreement.asp?EntityID $=4388>$

Wilkinson, R. and Marmot, M. 2003. Social Determinants of Health: The Solid Facts, 2nd edition, World Health Organization, Copenhagen.

World Health Organization (WHO) 2006. Constitution of the World Health Organization 2006, viewed 31 January 2011, available at <www.who.int/ governance/eb/who_constitution_en.pdf> 